\title{
Blade Crack Detection of Centrifugal Fan Using Adaptive Stochastic Resonance
}

\author{
Bingbing $\mathrm{Hu}$ and Bing $\mathrm{Li}$ \\ State Key Laboratory for Manufacturing Systems Engineering, Xian Jiaotong University, Xi'an 710049, China \\ Correspondence should be addressed to Bing Li; bli@mail.xjtu.edu.cn
}

Received 25 April 2014; Revised 5 October 2014; Accepted 6 October 2014

Academic Editor: Didier Rémond

Copyright ( 92015 B. Hu and B. Li. This is an open access article distributed under the Creative Commons Attribution License, which permits unrestricted use, distribution, and reproduction in any medium, provided the original work is properly cited.

\begin{abstract}
Centrifugal fans are widely used in various industries as a kind of turbo machinery. Among the components of the centrifugal fan, the impeller is a key part because it is used to transform kinetic energy into pressure energy. Crack in impeller's blades is one of the serious hidden dangers. It is important to detect the cracks in the blades as early as possible. Based on blade vibration signals, this research applies an adaptive stochastic resonance (ASR) method to diagnose crack fault in centrifugal fan. The ASR method, which can utilize the optimization ability of the grid search method and adaptively realize the optimal stochastic resonance system matching input signals, may weaken the noise and highlight weak characteristic and thus can diagnose the fault accurately. A centrifugal fan test rig is established and experiments with three cases of blades are conducted. In comparison with the ensemble empirical mode decomposition (EEMD) analysis and the traditional Fourier transform method, the experiment verified the effectiveness of the current method in blade crack detection.
\end{abstract}

\section{Introduction}

Centrifugal fan plays an important role in industrial applications at present. With rapid development of science and technology, centrifugal fan is growing larger, more precise, and more automatic in modern industry which also leads to the difficulty of detecting its potential faults caused by harsh working environment at unscheduled time. Once this fault occurs, it will cause great economic loss. Hence, it is necessary to ensure the availability and reliability of centrifugal fan. Although damage can occur on any part of the centrifugal fan, the most common type is blade damage. Statistics have shown that blade damage is generally susceptible to failure compared to other components. Blade failure can be caused by fatigue, excessive stress, load, and environmental factors. Thus, special attention should be paid to the structural health of blades because blades are one of the most critical components in centrifugal fan.

As an efficient tool, vibration analysis has attracted considerable interest and has been widely applied to fault diagnosis of mechanical equipment in the past decades, since vibration signals carry a great deal of information representing mechanical equipment condition. If there is a crack in the blade, it will result in abnormal blade vibration. The vibration information is a typical characteristic for blade fault diagnosis. When an impeller rotates, air, the communication medium between the blade and the casing, can deliver blade vibration information on the casing for air inside centrifugal fan and will glide on the blades resulting in a dynamic pressure difference. Based on vibration signals, different methods of blade crack detection have been investigated by many researchers. Rao and Dutta investigated blade crack condition classification by using characteristic vibration signal information for assessing health of compressor blades in two operating plants [1]. Witek experimented with the helicopter turbo-engine compressor blades and analyzed crack propagation process of gas turbine blades with the help of vibration signals to recognize blade cracks [2]. By analyzing the acquisition data of the wind turbine, Yan and Xu calculated the correlation dimension of blades' vibration signals and used the method to classify the difference at working state of blades effectively [3]. Gubran and Sinha investigated the distinct difference between the healthy and the faulty blade conditions via the measured shaft torsional vibration through experiments [4]. Most of the above research is focused on gas turbine blades; however, the study on blade crack detection 
of centrifugal fan is rare, despite the similarities between the two kinds of machines. Therefore, it is very important to carry out experiments on centrifugal fan test rigs.

Conventional signal processing techniques including time-domain statistical analysis and Fourier transform are most commonly used methods for signal analysis and fault diagnosis. Kuo investigated the diagnosis of the loose blades using Fourier analysis undertaken on the measured vibration signals [5]. Barragan reported that the comparison between vibration fast Fourier transform (FFT) patterns and a library of known vibration induced patterns of engine faults enabled the detection of mechanical faults that included foreign object damage, blade loss part, blade rub, and loose joints [6]. Based on the vibration spectrum analysis of torsional vibration signals, nonintrusive measurement method was reported to be effective in the diagnosis and detection of changes in blade natural frequency with blade cracks being amenable to detection [7-9]. Since these studies are based on the assumption that the process generating signals is stationary and linear, further feature extraction methods are urgently needed for blade fault diagnosis.

As a new time-frequency analysis method, empirical mode decomposition (EMD) based on the local characteristic time scales of a signal may decompose the complicated signal into a set of complete and almost orthogonal components named intrinsic mode function (IMF) [10]. To alleviate the problem of mode mixing in EMD, ensemble empirical mode decomposition (EEMD), an improved method of EMD, is presented by $\mathrm{Wu}$ and Huang [11] recently. Yang et al. used the EMD method to analyze the feature intensity level power signals which are measured through the terminals of 3-phase wind turbine induction generator [12]. In another study, bivariate empirical mode decomposition (BEMD) was used to detect both incipient and electrical faults by Yang et al. [13]. Abouhnik and Albarbar put forward a novel approach called empirically decomposed feature intensity level (EDFIL) to reveal the effects of the blade faults [14]. Lei et al. proposed a new EEMD-based method for fault diagnosis of rotating machinery and the method was applied to rub-impact fault diagnosis of a power generator and early rub-impact fault diagnosis of a heavy oil catalytic cracking machine set [15]. Although the EEMD method is an improvement of EMD method, its problems such as the use of splines in the interpolation procedure, the calculation of mean value, and signal end effects in the EEMD method need to be solved. In addition, it is very difficult to select the appropriate magnitude of noise and number of ensembles.

Stochastic resonance (SR) proved to be an effective tool in processing signals with low signal-to-noise ratio by utilizing noise to enhance signal weak characteristics [16]. Adaptive stochastic resonance (ASR) was first introduced by Mitaim and Kosko in his study on the SR optimality conditions [17]. It can be seen as an improvement of the SR. Based on SR or ASR unique advantage of using noise to enhance weak signals instead of eliminating noise, its application to mechanical fault diagnosis has been studied extensively [18-29]. It is very difficult to detect blade fault feature by means of conventional signal processing techniques since the blade crack vibration signal is very weak due to strong noise interference in the practical working centrifugal fan. Therefore, ASR method can be applied to characteristic signal detection of blade cracks because it can enhance weak signal feature to some extent.

This paper applies the weak vibration signals collected by ASR method to blade crack detection. Experiments are carried out to verify the effectiveness of this method in a test rig. The paper is organized as follows. The theory of characteristic extraction for blade crack is introduced in Section 2. Section 3 describes the experimental setup for blade crack monitoring. Then experimental studies on two cases of cracked blade and normal blade signals are conducted to confirm the effectiveness of the ASR method for extracting weak feature frequency in comparison with traditional Fourier transform and EEMD methods in Section 4. Finally, conclusions are drawn in Section 5.

\section{Theoretical Background}

2.1. SR Theory. SR is an effective nonlinear method in weak signal analysis, by which the weak signal is enhanced, and the noise is weakened through the interaction of a nonlinear dynamic system, a small parameter signal, and noise. In other words, noise can be used to enhance the weak signal by means of a nonlinear dynamical system.

Three ingredients are needed to produce a SR phenomenon: (1) a bistable or multistable system, (2) a weak coherent input (a periodic or aperiodic signal), and (3) a source of noise that is inherent in the system or adds to the coherent input [30]. The bistable system, as a common SR model, is often adapted to investigate SR phenomenon, which is described by the nonlinear Langevin equation as follows:

$$
\frac{d y}{d x}=-U^{\prime}(x)+A \cos \left(2 \pi f_{0} t+\varphi\right)+n(t),
$$

where $U(x)$ denotes the reflection-symmetric quartic potential as follows:

$$
U(x)=-\frac{a}{2} x^{2}+\frac{b}{4} x^{4}
$$

Let $n(t)=\sqrt{2 D} \xi(t)$ with $\langle n(t), n(t+\tau)\rangle=2 D \delta(t)$, where $D$ is the noise intensity and $\xi(t)$ represents a Gaussian white noise with zero mean and unit variance.

Then, (1) can be written as

$$
\frac{d y}{d x}=a x-b x^{3}+A \cos \left(2 t f_{0} t+\varphi\right)+\xi(t),
$$

where $a$ and $b$ are barrier parameters, which are positive real parameters. $A$ is the periodic signal amplitude and $f_{0}$ is the driving frequency.

According to (2), the potential function $U(x)$ is symmetrical and has two stable fixed points at $x_{m}= \pm \sqrt{a / b}$ and one quasistable fixed point at $x=0$. A potential barrier separates the two stable fixed points with the height given by $\Delta U=a^{2} / 4 b$. Figure 1 shows the bistable state function curve when the parameter is $a=b=1, A=0$. In the absence of the periodic input signal and noise, the potential 


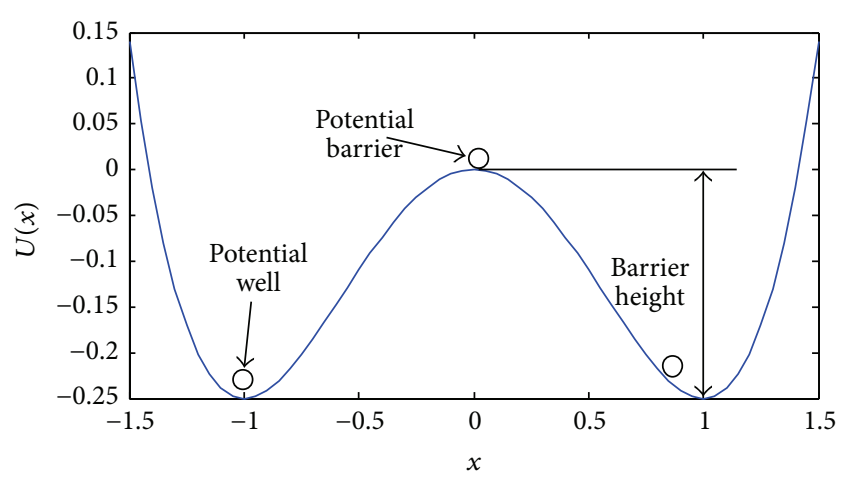

FIgURE 1: The bistable state function curve $(a=b=1, A=0)$.

function $U(x)$ is determined by the initial conditions and never changes. Only in the presence of the periodic input signal can the potential barrier be reduced and the particle, which stays in one potential well, may surmount the energy barrier and enter another potential well. In fact, the nonlinear system output $x(t)$ is the Brownian particle trajectory in the potential function $U(x)$ under the interaction of the periodic input signal and the noise.

Based on the above analysis, the potential function $U(x)$ plays a key role in the occurrence of the SR phenomenon, while $U(x)$ is jointly decided by system parameters $a$ and $b$. That is to say, a pair of appropriate system parameters $a$ and $b$ are able to induce the SR phenomenon well. Therefore, it is important to set and optimize the best combination of system parameters $a$ and $b$ in SR.

2.2. ASR Method. In this paper, an adaptive stochastic resonance method is used to process blade crack vibration signal and to extract weak fault characteristics in the experiments. The grid search method is used to automatically search and optimize SR parameters $a$ and $b$. This method can systematically search all of the possible states. If the required maximum is known to be within a finite area defined by upper and lower bounds of each of the independent variables, then the grid search method can be applied. For this purpose, one must set up a grid over the area of interest and evaluate the objective function at each node of the grid. At last, a maximum can be found between the guidelines after the computation of the objective function values in all the nodes of the grid [31]. In this paper, according to practical application and experiment results analysis, the search band of system parameters and the step length depend on mainly the following two points. (1) Using a single parameter adjustment method, make $b=1$, and the initial search range of $a$ is $[0.01,2]$, which is adjustable according to the calculation results. (2) Considering the result accuracy and computational efficiency, the search step length can be adjusted according to the range of the system parameters. When the range is large, appropriately increase the step length; when the range is small, appropriately reduce the step length, which is useful in finding the optimal solution, where the initial search step is 0.02 . To solve large parameters signals feature extraction by ASR method in engineering application, frequency-shifted and rescaling technique are introduced and demonstrated in [32], which is adopted to preprocess the input signals.

The objective function in the optimization process of grid search method is to select the signal-to-noise ratio of the output signal of SR. It is known to be easy to recognize the signal when the signal frequency amplitude is the maximum in FFT frequency spectra. In $[33,34]$, if the location of the highest spectral peak is recorded in an iteration process in which one of the system parameters is varied and the other is fixed, the relationship between the varied system parameter and the location of the highest spectral peak can be established graphically. In the graph, the height of one horizontal segment represents the frequency value of interest only if the changing range of parameters covers the best optimal parameters. However, since more than one horizontal segment may exist, the highest spectral peak location may present more than one possible frequency value. We can get more reasonable system parameters by combining the highest spectrum peak and the signal-to-noise ratio as an optimization index. Therefore, if the input signal frequency value cannot be estimated exactly, the maximum value in the output spectra instead of the input signal frequency amplitude may be used to calculate the SNR of the output signal.

Then, $X(k)$ is the discrete Fourier transform of the signal $x=\left\{x_{1}, x_{2}, \ldots, x_{N}\right\}, N$ is the length of the signal, and $Y(k)$ is the amplitude sequence in frequency domain. Consider

$$
\begin{aligned}
& X(k)=\sum_{n=1}^{N} x(n) e^{-j 2 \pi(k-1)(n-1) / N}, \quad 1 \leq k \leq N, \\
& Y(k)=\frac{2|X(k)|}{N}, \quad 1 \leq \frac{N}{2},
\end{aligned}
$$

where $k_{0}$ and $f_{\max }$ are defined as the highest spectrum peak position and its corresponding spectral frequency in spectra of the signal, respectively. Consider

$$
k_{0}=\frac{f_{\max } \times N}{f_{s}}+1
$$

Here, $f_{s}$ is sample frequency. And then, the SNR in this paper can be calculated as follows:

$$
\mathrm{SNR}=\log 10 \frac{Y\left(k_{0}\right) \times(N / 2)}{\sum_{k=1}^{N / 2} Y(k)-Y\left(k_{0}\right)} .
$$

Figure 2 shows the flow chart of the ASR method which consists of the following procedural steps. (1) The initial values and the objective function of the grid search method are set based on the SNR. (2) To meet the requirement of small parameters of SR, the frequency-shifted and rescaling technique are adopted to preprocess the vibration signals collected from the centrifugal fan test rig. (3) The grid search method is employed to adaptively select and optimize SR parameters $a$ and $b$. (4) In terms of the objective function, the best one is saved by comparing different combinations of parameters $a$ and $b$. (5) The best combination is assigned to SR and the ASR system developed. (6) The weak features submerged in the vibration signal of the impeller are 


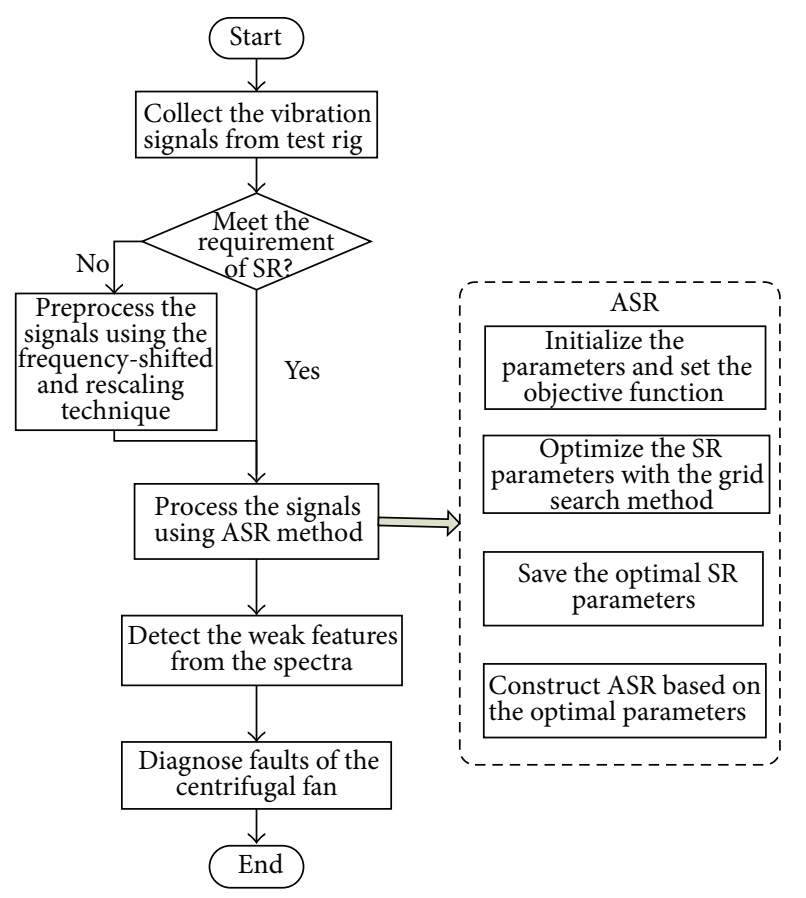

FIGURE 2: Flow chart of the ASR method.

extracted using the ASR method. (7) The blade crack in centrifugal fan is eventually diagnosed according to the detected characteristics.

2.3. Blade Passing Frequency. Blade passing frequency, as a high-frequency component, has proved to be a good feature in health diagnosis of the blades $[35,36]$. It is the main source of centrifugal fan noise and can be applied to estimate blade health condition. The blade passing frequency, the frequency at which the blades pass a fixed position, is obviously the number of blades multiplied by the rotational speed.

When a rotating blade vibrates, the amplitude of blade passing frequency will show significant variation. In other words, the vibration amplitude of blade passing frequency is very sensitive to operating condition of the blade. Tuned blades usually do not show vibration signals during operation. But when a blade has cracks, the amplitude of blade passing frequency will deviate from the normal value. It can be seen that the low-frequency components related to blade vibration may be modulated to blade passing frequency during blade rotation. If unbalanced rotor conditions exist in the experiments, the shaft frequency is also modulated to blade passing frequency. However, the sideband frequency produced by blade cracks is different from shaft frequency which does not mean there is a blade with cracks if shaft frequency is the sideband frequency for blade passing frequency. Only in the experiments can the sideband frequency produced by blade cracks be used to determine the modulated characteristic frequency. Because the information produced by blade cracks is very weak and overwhelmed by noise, it is very difficult to extract feature frequency. To better extract feature information, the ASR method is used to process blade

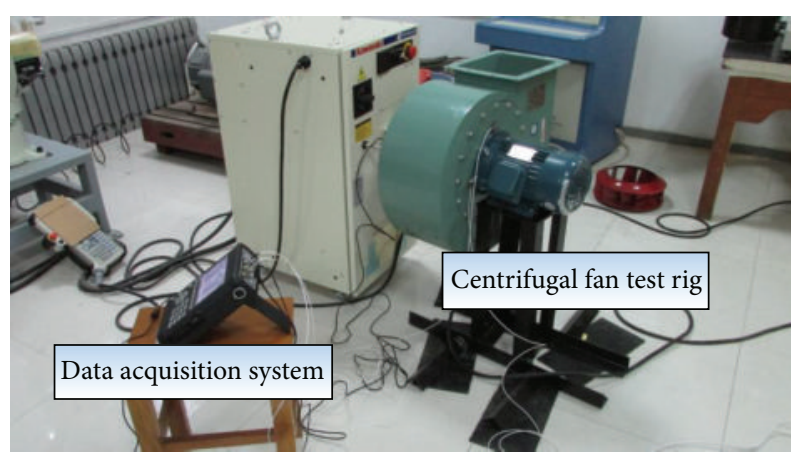

FIgURE 3: Centrifugal fan blade crack test rig.

vibration signals and is helpful to improve the recognition for blade crack.

\section{Experimental Test}

3.1. Test Rig. To verify the effectiveness of the ASR in fault diagnosis of centrifugal fan blade crack, a test rig of signal analysis based on bearing seat vibration is developed and experiments on it are conducted. The experimental rig is shown in Figure 3, which principally includes a three-phase electric machine, bearing seats, impeller with crack and no crack, support pedestal, and data acquisition system. The three-phase electric machine is the power input of centrifugal fan and its highest speed can reach $2950 \mathrm{rpm}$. In this experiment, the speed of the impeller is $2950 \mathrm{rpm}$ under different conditions. The experiment was carried out under two conditions: normal and crack conditions. The crack length during the experiment is $32 \mathrm{~mm}$ or $16 \mathrm{~mm}$. The SF (shift frequency) and blade passing frequency correspond to $49 \mathrm{~Hz}$ and $490 \mathrm{~Hz}$, respectively.

3.2. Data Acquisition. The location of the vibration sensor is important as it is directly related to the accuracy of fault diagnosis. If the vibration sensor position is far from the blade crack, it is difficult to classify blade crack using vibration signal analysis [37]. Figure 4 displays sensor installation position and impeller with crack in the experiment. A crack was made through steel saw blade to simulate blade crack fault. The crack, which is located at the end of inlet of centrifugal fan, was of length $16 \mathrm{~mm}$ or $32 \mathrm{~mm}$, and all had a consistent $2 \mathrm{~mm}$ width and $1 \mathrm{~mm}$ depth. The acceleration sensor was located at the bearing seat from the driving end to monitor vibration signals which corresponded to the crack location in the axial direction. In order to ensure the rationality of the results, blade vibration experiments with no crack and with crack were conducted, respectively. The accelerometer was produced by KISTLER Electronics factory. The sensitivity was $102.8 \mathrm{mv} / \mathrm{g}$. The vibration signal was acquired by COCO 80 system under two conditions: normal and crack conditions. The data sampling frequency was $5120 \mathrm{~Hz}$ for vibration signals. 


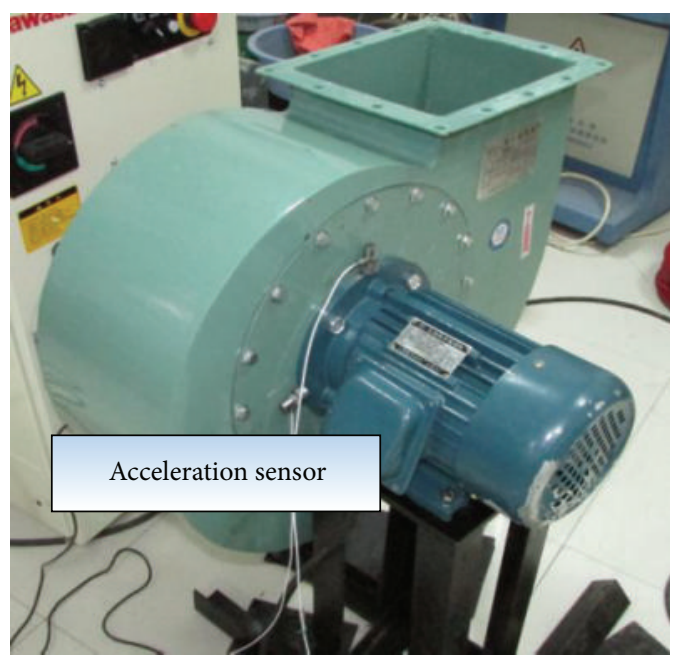

(a)

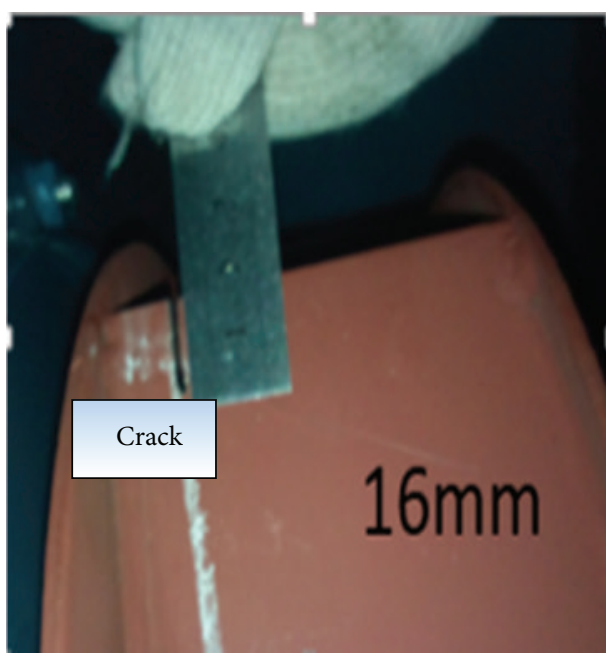

(b)

FIGURE 4: (a) Sensor installation position and (b) centrifugal fan with cracked blade.

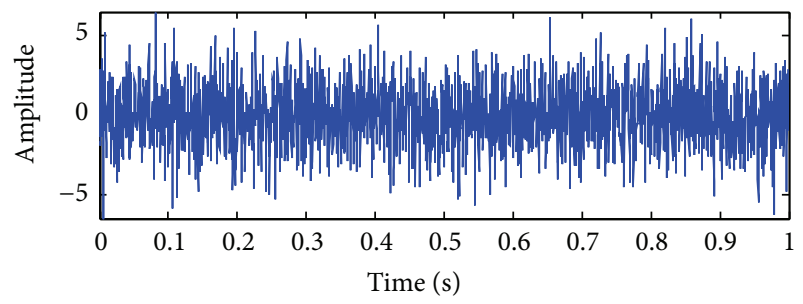

(a)

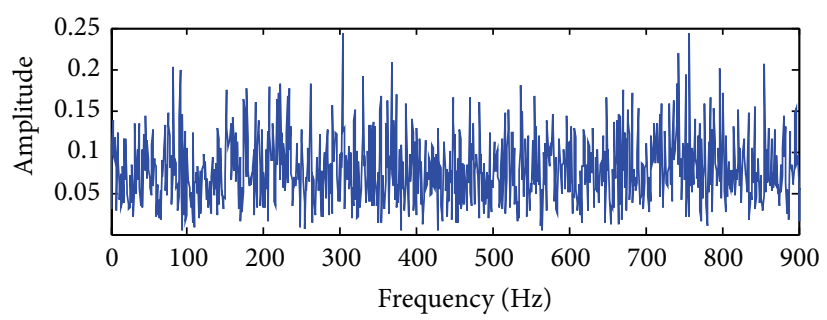

(b)

FIGURE 5: Simulation signal: (a) time-domain waveform and (b) frequency spectrum.

3.3. Simulation Experiments. To validate the usefulness of the ASR method in extracting weak signals, a simulation experiment is carried out. The simulated signal consists of a sin wave embedded in heavy noise. The sampling frequency is $2048 \mathrm{~Hz}$, and the length of the simulated signal is 2048 points. The amplitude $A$ and the frequency $f$ of the sin wave are 0.17 and $50 \mathrm{~Hz}$, respectively, and the intensity $D$ of the noise equals 2. It can be seen from Figure 5(a) that the waveform of the simulated signal is corrupted with strong noise and cannot be distinguished. Figure 5(b) shows the frequency spectrum of the simulated signal. In the frequency spectrum, the weak characteristic frequency of $50 \mathrm{~Hz}$ is completely swamped by the heavy noise. The proposed ASR method is used to process the simulated signal and its output signal is obtained. In signal processing, the parameters are set as follows: $a=0.87, b=1$, the rescaling ratio $R=200$, the pass-band cutoff frequency $F_{p}=46 \mathrm{~Hz}$, the stop-band cutoff frequency $F_{s}=44 \mathrm{~Hz}$, and the carrier frequency $f_{c}=44 \mathrm{~Hz}$. Figures 6(a) and 6(b) show the time-domain waveform and frequency spectrum of the output signal, respectively. After analyzing the frequency spectrum of the output signal, we find that the frequency $50 \mathrm{~Hz}$ can be clearly detected.

In comparison, the simulation signal is analyzed again by EEMD method with the parameters that the number of ensembles is 100 and the standard deviation of the added
TABLE 1: Characteristic frequencies for test rig.

\begin{tabular}{lccc}
\hline $\begin{array}{l}\text { Speed } \\
(\mathrm{RPM})\end{array}$ & $\begin{array}{c}\text { Number of } \\
\text { blades }\end{array}$ & $\begin{array}{c}\text { Shaft frequency } \\
(\mathrm{Hz})\end{array}$ & $\begin{array}{c}\text { Blade pass } \\
\text { frequency }(\mathrm{Hz})\end{array}$ \\
\hline 2950 & 10 & 49 & 490 \\
\hline
\end{tabular}

white noise is 0.02 , and the decomposition result is displayed in Figure 7. It is clear that the EEMD method fails to provide the reasonable decomposition because it is very difficult to select the appropriate magnitude of noise and number of ensembles. We cannot find the sin wave and the characteristic $50 \mathrm{~Hz}$ in each IMF and its frequency spectrum. Thus, the processing result with the ASR method is better than that of the EEMD method.

\section{Analysis of Experimental Results and Discussions}

4.1. Experimental Results. In this section, the ASR method is applied to diagnosing faults of blade crack. As introduced in Section 3, an experiment with normal and crack conditions is used to prove the validity of the method in the test rig. The vibration signals are collected at the highest speed. The related characteristic frequencies are summarized in Table 1. 


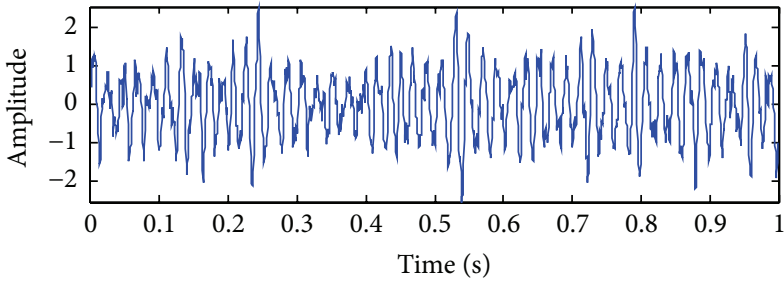

(a)

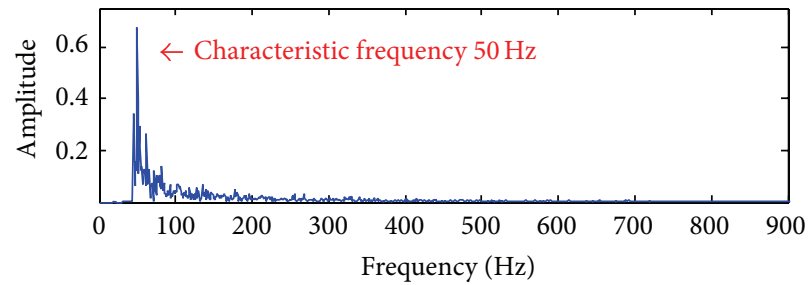

(b)

FIGURE 6: Result of the ASR method for the simulation signal: (a) output signal and (b) frequency spectrum of the output signal.
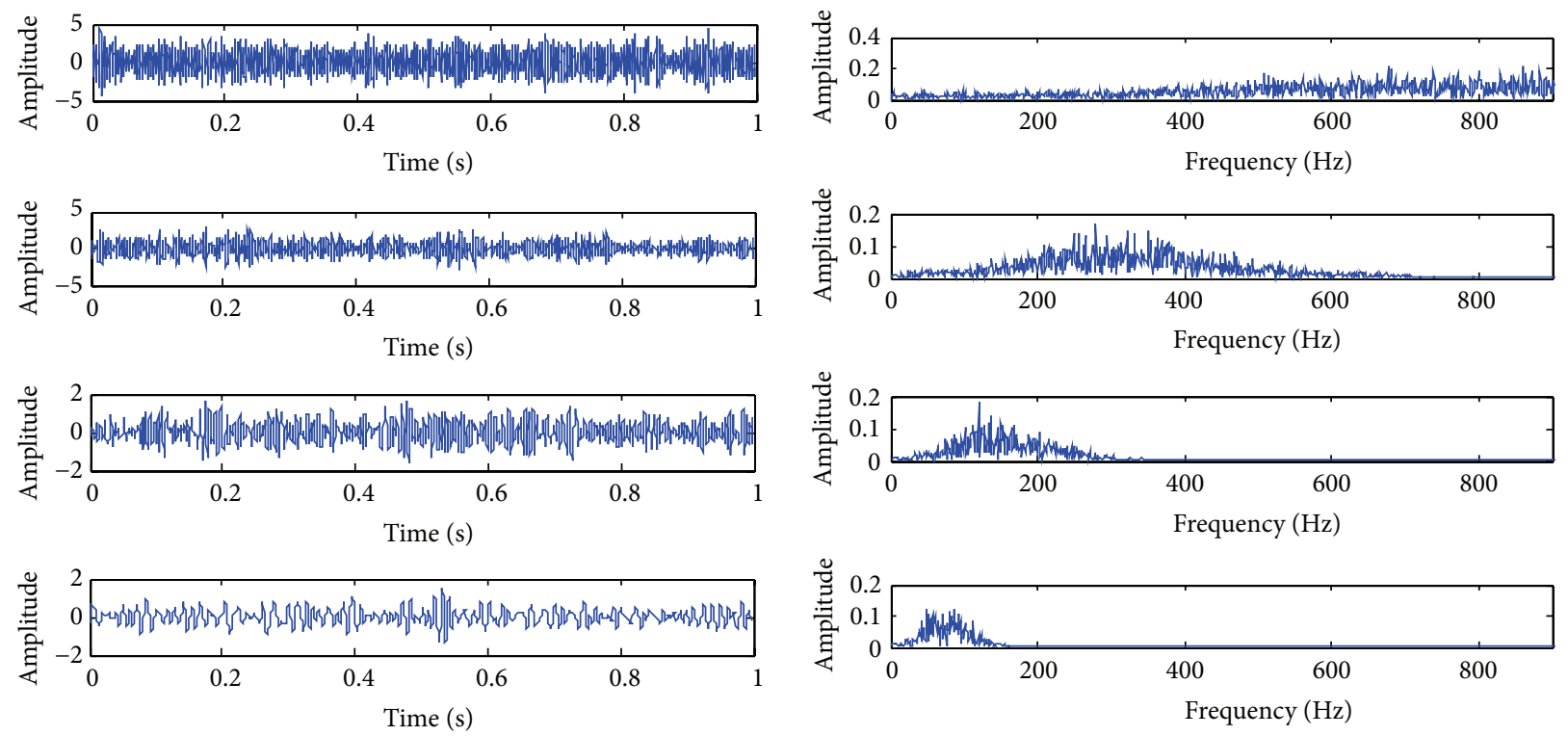

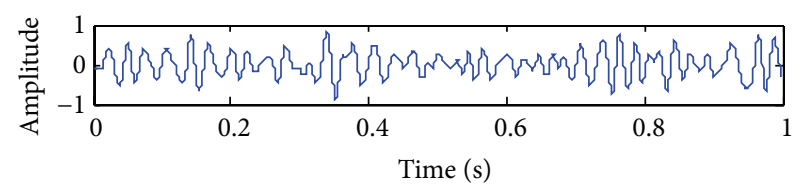
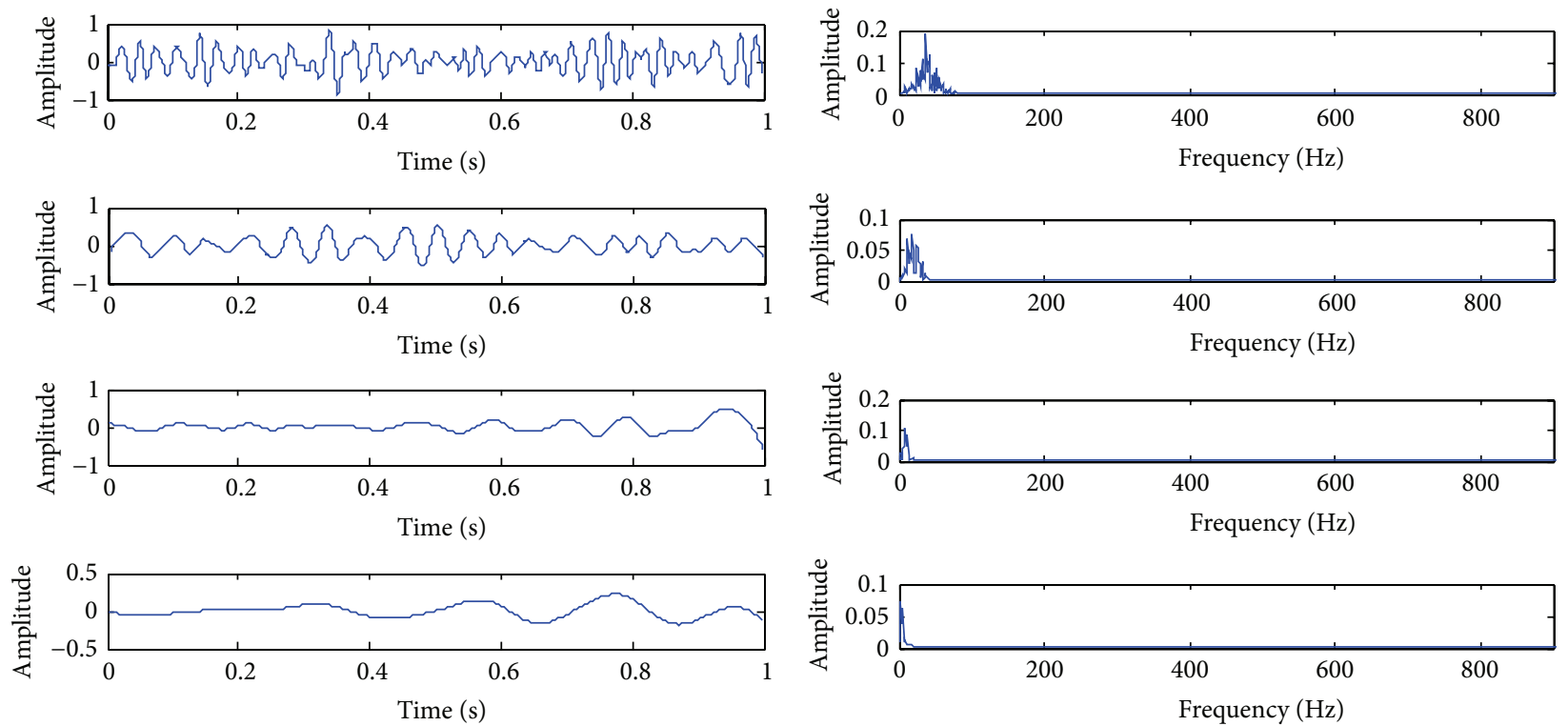

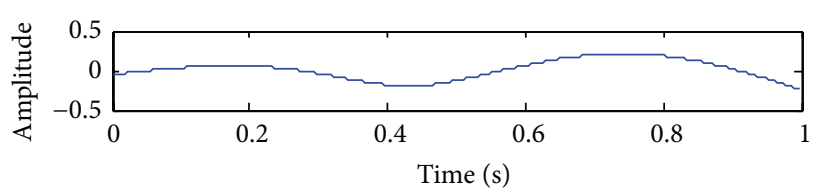

(a)

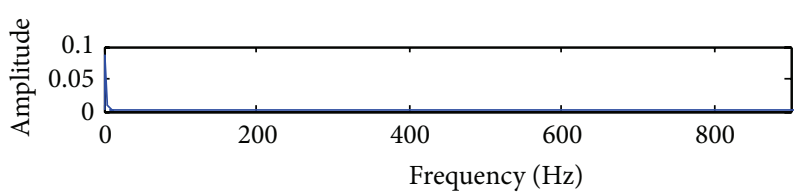

(b)

FIGURE 7: Result of the EEMD method for the simulation signal: (a) IMFs and (b) frequency spectra of IMFs. 


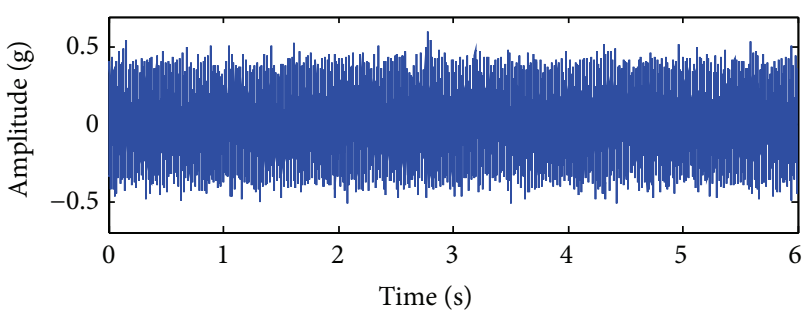

(a)

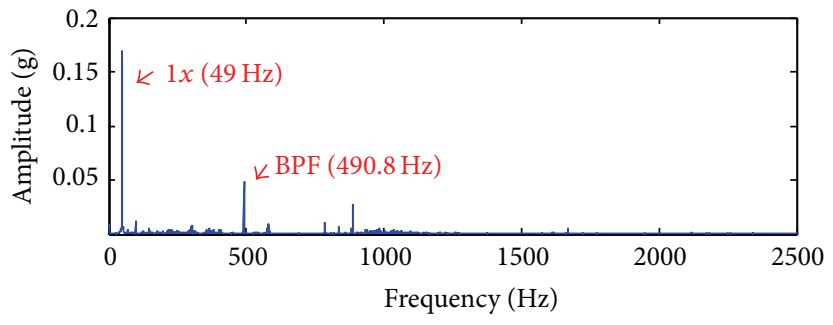

(b)

FIGURE 8: Original vibration signals under $32 \mathrm{~mm}$ crack condition: (a) time-domain waveform and (b) frequency spectrum.

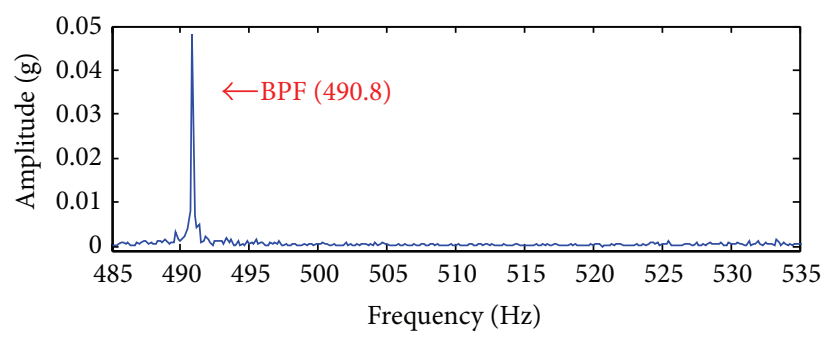

FIGURE 9: Zoomed-in spectrum under $32 \mathrm{~mm}$ crack condition.

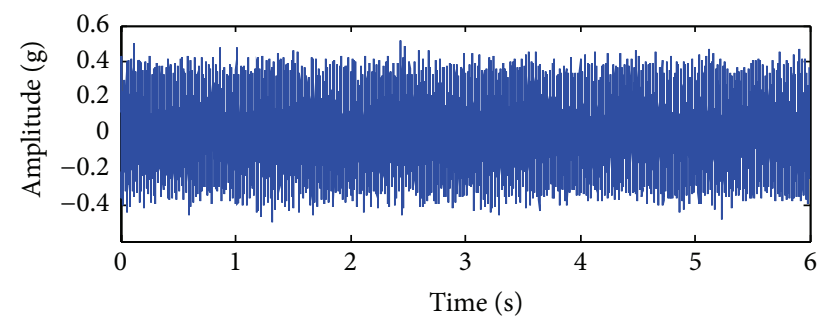

(a)

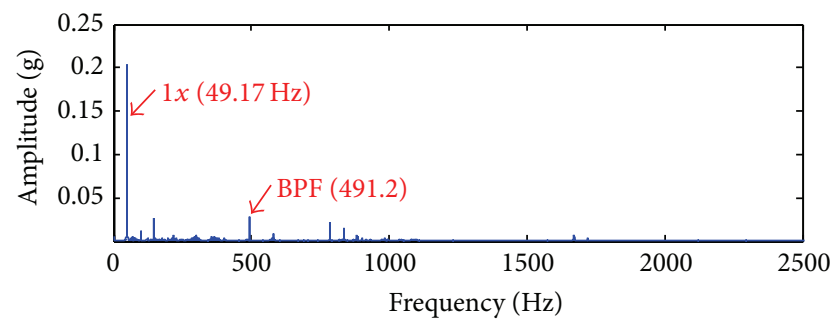

(b)

FIGURE 10: Original vibration signals under normal condition: (a) time-domain waveform and (b) frequency spectrum.

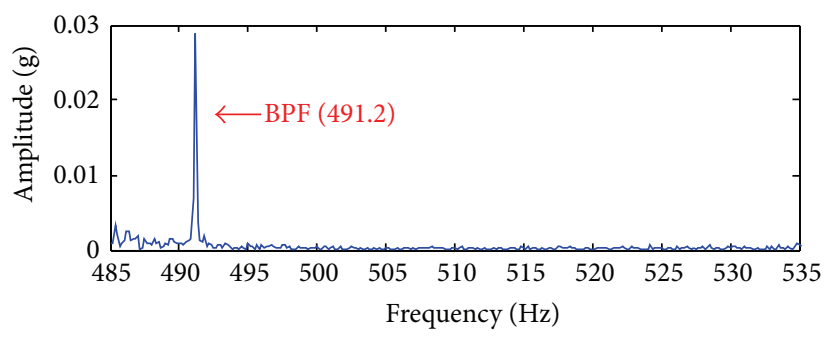

FIGURE 11: Zoomed-in spectrum under normal condition.

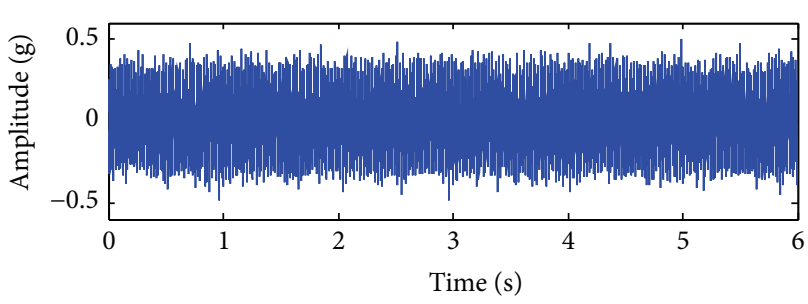

(a)

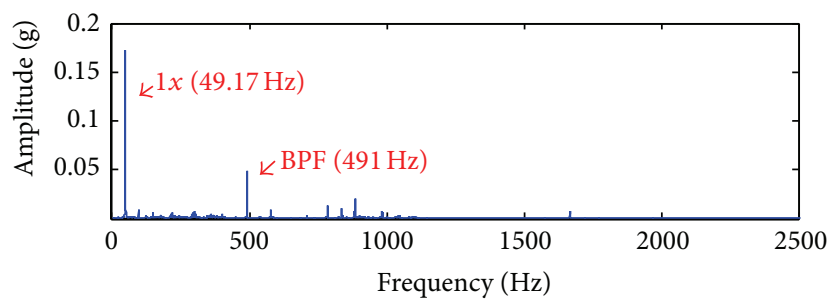

(b)

FIGURE 12: Original vibration signals under $16 \mathrm{~mm}$ crack condition: (a) time-domain waveform and (b) frequency spectrum. 


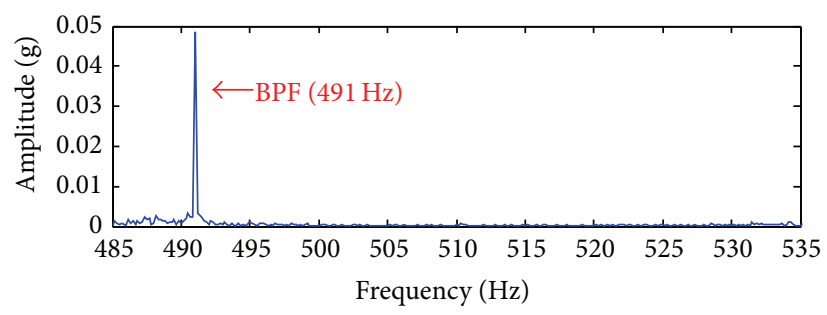

FIGURE 13: Zoomed-in spectrum under $16 \mathrm{~mm}$ crack condition.

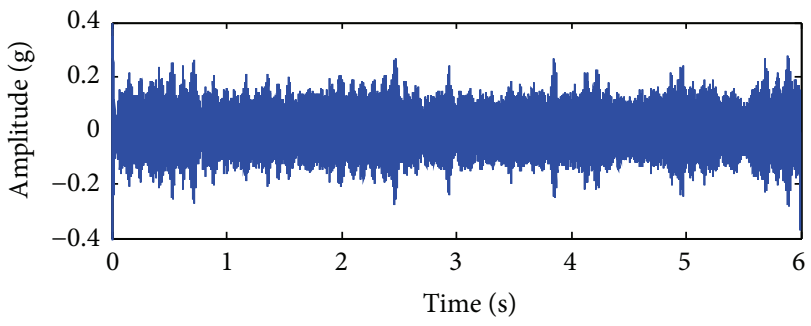

(a)

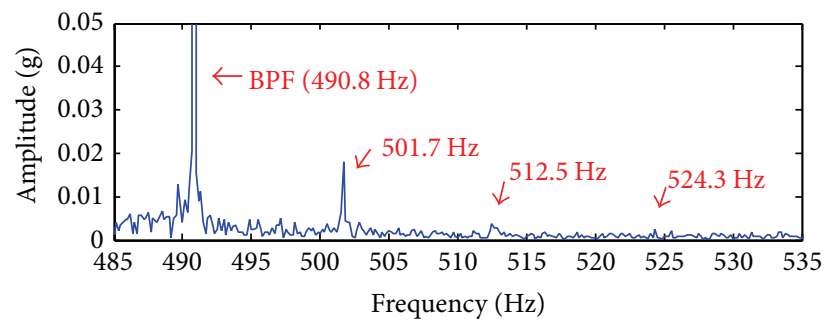

(b)

FIGURE 14: Output signals of the ASR method under $32 \mathrm{~mm}$ crack condition: (a) time-domain waveform and (b) frequency spectrum.

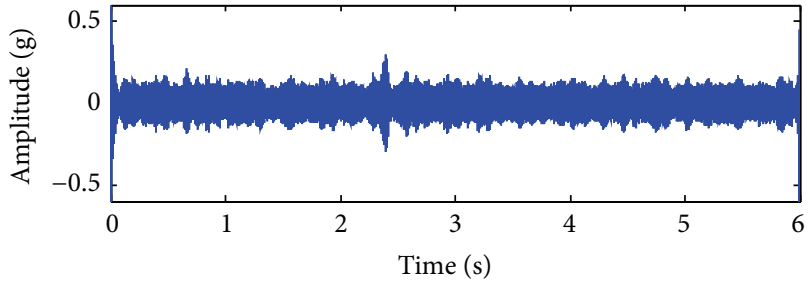

(a)

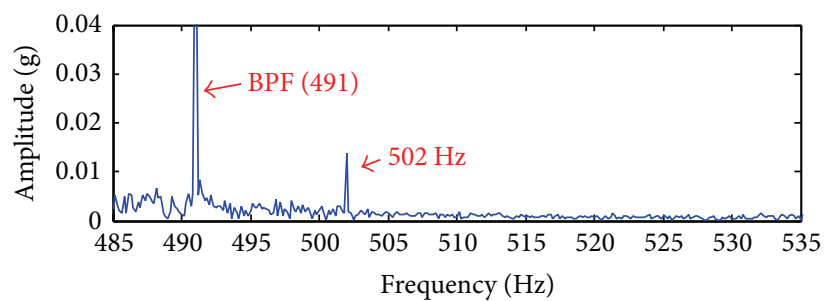

(b)

FIGURE 15: Output signals of the ASR method under $16 \mathrm{~mm}$ crack condition: (a) time-domain waveform and (b) frequency spectrum.

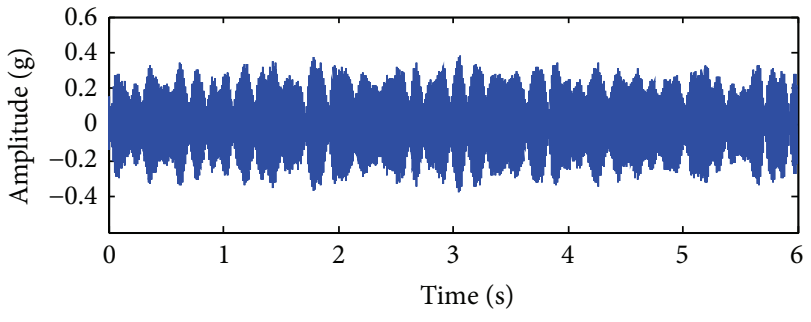

(a)

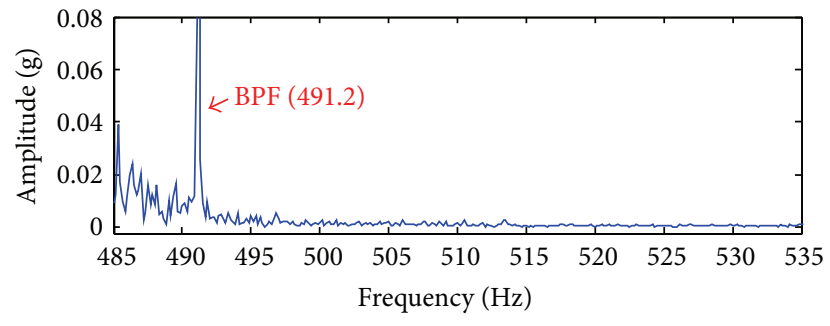

(b)

FIGURE 16: Output signals of the ASR method under normal condition: (a) time-domain waveform and (b) frequency spectrum.

Figures 8(a), 10(a), and 12(a) show the vibration signals collected at the motor speed of $2950 \mathrm{rpm}$, which represent three cases of the normal impeller and two crack impellers with $32 \mathrm{~mm}$ and $16 \mathrm{~mm}$ in the depth, respectively. As observed from the time-domain waveforms of the vibration signals, there is no clear difference in the three cases. Their amplitudes are basically the same. Time domain cannot provide clear characteristics for the blade crack as the collected signals are very weak. In other words, weak signals are submerged in heavy noise. Therefore, it is difficult to extract the crack information under normal and crack conditions just according to time-domain information.

In order to identify the fault information with vibration signals, FFT is applied to signal processing for crack detection in the experiments. Frequency domain vibration signals under the normal and crack conditions are shown in Figures 8(b), 10(b), and 12(b), respectively. After analyzing the frequency spectra of the three cases, we find that the shaft frequency and its harmonics are quite dominant. But the blade passing frequency we need is very weak in the frequency spectra. To verify the effectiveness for sideband 


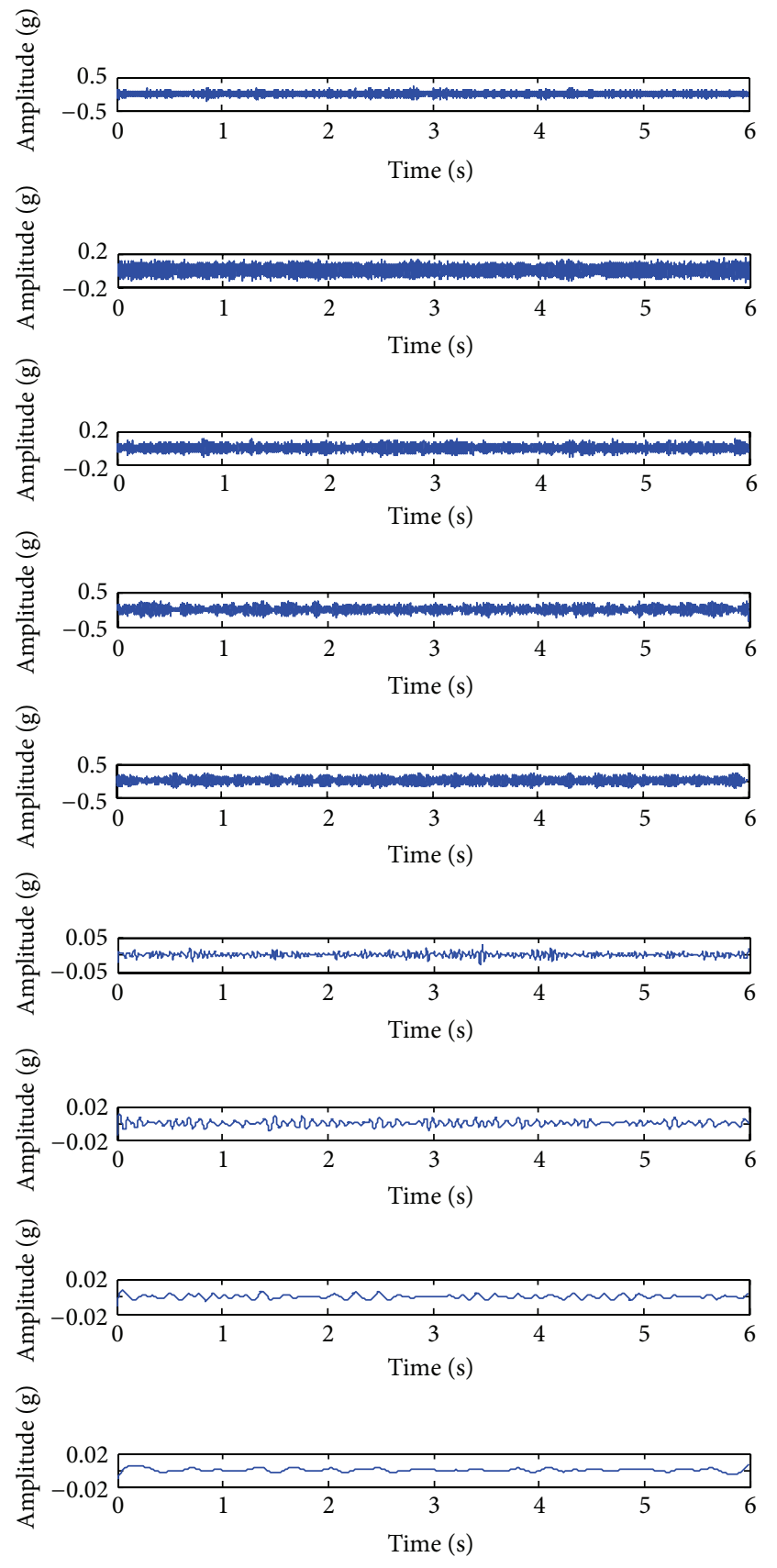

(a)
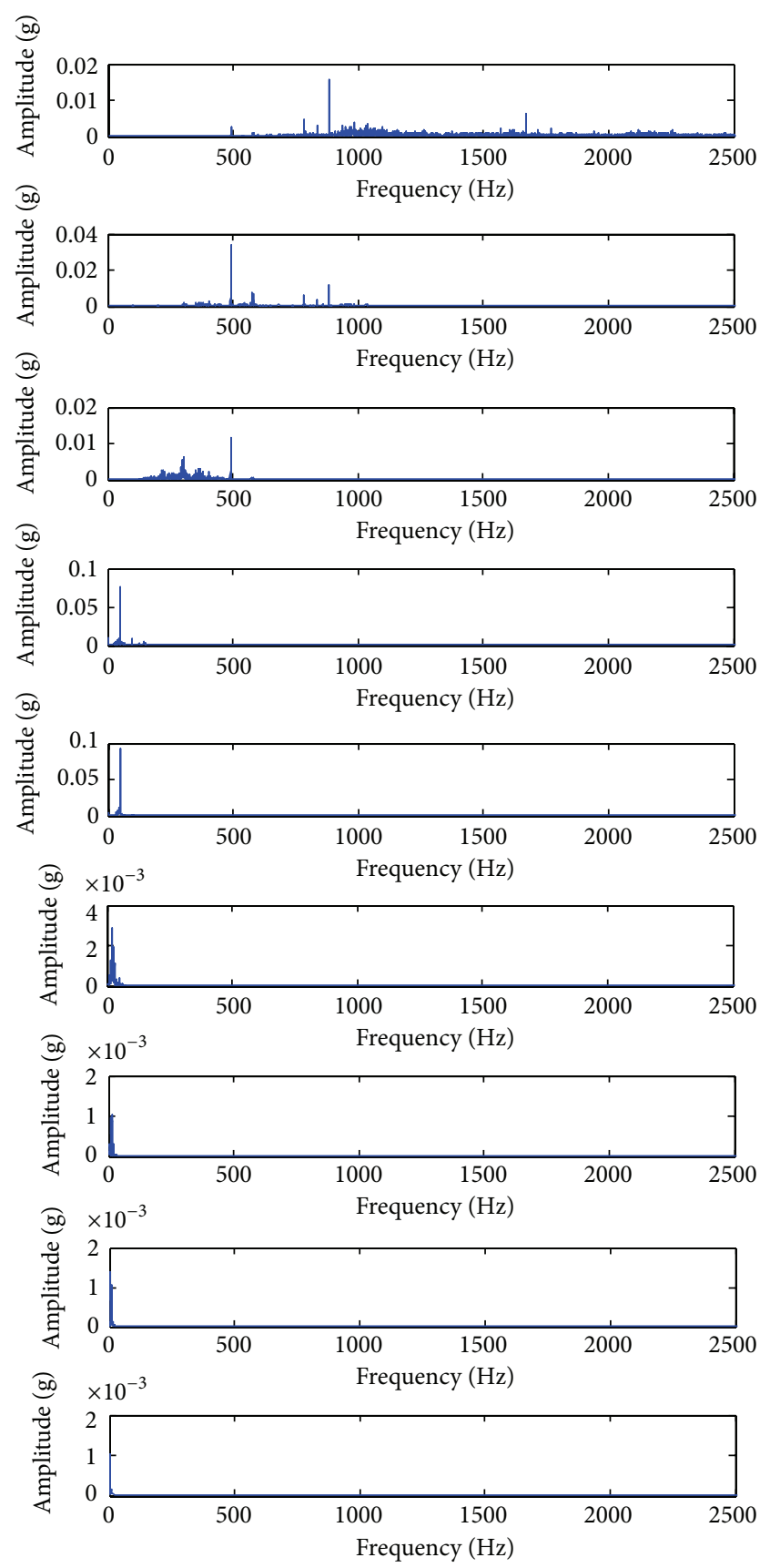

(b)

FIGURE 17: Results of the EEMD method under $32 \mathrm{~mm}$ crack condition: (a) IMFs and (b) frequency spectra of the IMFs.

frequency analysis, we zoom in the frequency spectra with respect to the blade passing frequency and present the zoomed-in spectra in Figures 9, 11, and 13, respectively. It is obvious that the sideband frequency is not clear due to noise interference. This implies that it is unfeasible to detect the fault characteristics of blade crack from both the vibration signals and their frequency spectra. Further investigation should be carried out for crack determination.

Then, the above three signals are processed using the ASR method. In the experiments, we select the scaling values $R=500$, the pass-band cutoff frequency $F_{p}=482 \mathrm{~Hz}$, the stop-band cutoff frequency $F_{s}=480 \mathrm{~Hz}$, and the carrier frequency $f_{c}=480 \mathrm{~Hz}$, under the condition of the three tests. The relative system parameters are $a=0.25, b=1$ under the $16 \mathrm{~mm}$ crack condition and $a=0.23, b=1$ under the $32 \mathrm{~mm}$ crack condition. Corresponding to the normal and crack conditions of the impeller, three output signals displayed in Figures 14(a), 15(a), and 16(a) are produced by the ASR method. Figures 14(b), 15(b), and 16(b) show their frequency spectra of the three output signals. Similar to the original vibration signals, it is obvious that the timedomain waveforms of the output signals cannot provide 

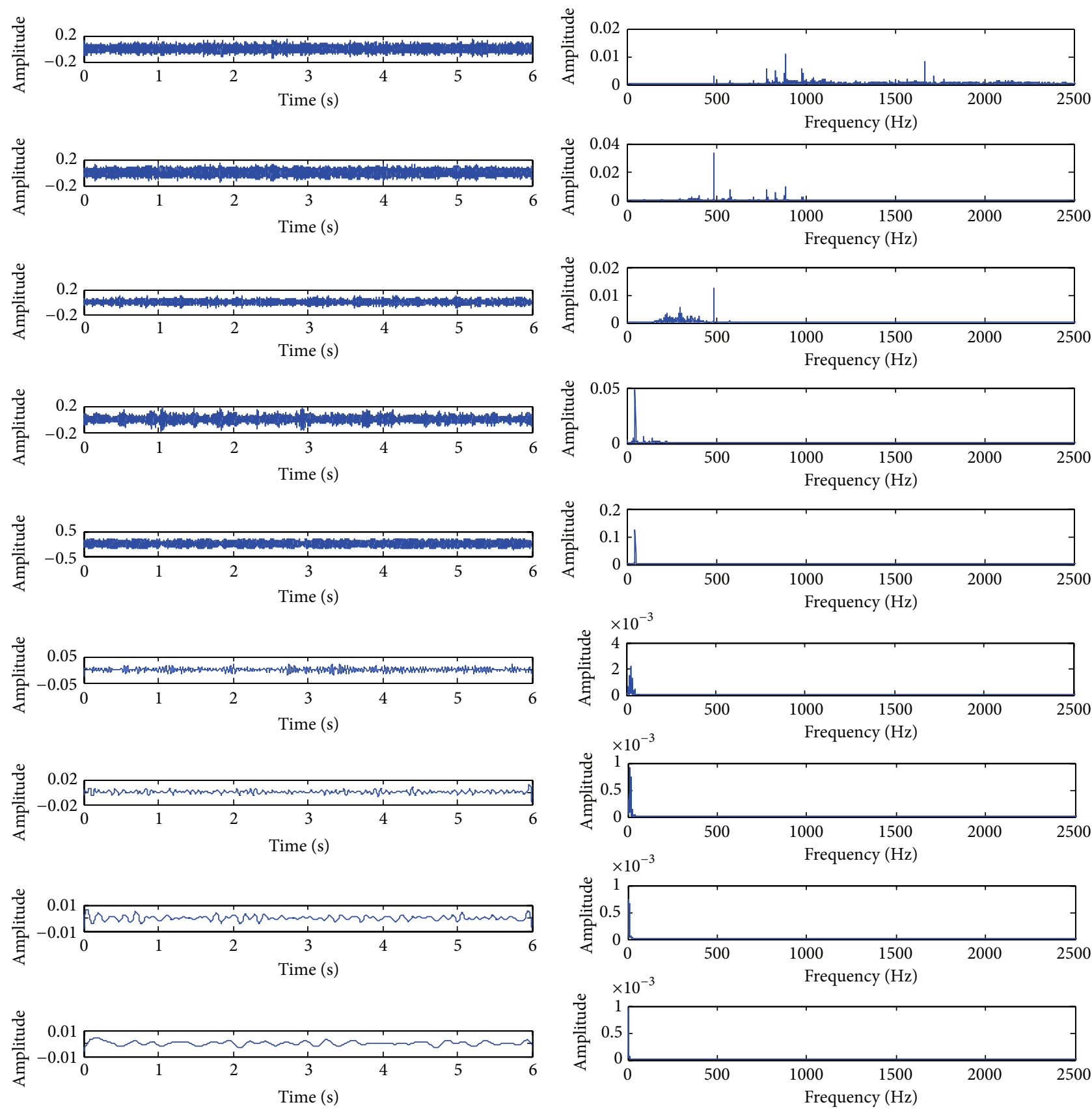

(a)

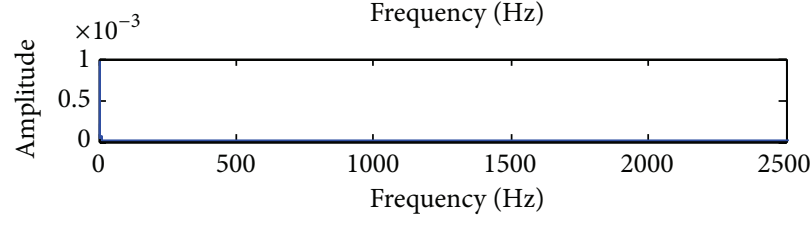

(b)

FIGURE 18: Results of the EEMD method under $16 \mathrm{~mm}$ crack condition: (a) IMFs and (b) frequency spectra of the IMFs.

useful information for diagnosing the blade crack either. However, we can clearly see that it is important that there are obvious frequency multiplications or harmonics for the crack conditions shown in Figures 14(b) and 15(b), because it means that the blade has cracks and is working under crack conditions. That is to say, key characteristics indicating the occurrence of blade crack faults in the experiments, such as the blade passing frequency, are revealed in the frequency spectra of the output signals. Compared with traditional time or frequency methods, it also verifies the effectiveness of the ASR method for crack detection.
In contrast, the EEMD method is also utilized to process the above vibration signals collected under normal and crack conditions. In signal processing, the parameters are set as follows: the number of ensembles is 100 and the standard deviation of the added white noise is 0.02 . The decomposition result is displayed in Figures 17 and 18. It is clear that the problem of mode mixing appears between different IMFs and there are serious distortions for each IMF. We cannot see the characteristic frequencies of blade crack for both crack conditions at all. This result shows that the EEMD method is not effective because it cannot provide reasonable 
decomposition when the signals extracted from the cracked blade are too weak. Thus, it is demonstrated that the proposed ASR method is better than the EEMD method in discovering weak characteristic for centrifugal fan fault diagnosis.

4.2. Discussion. (1) From the results of the simulation and actual experiments, it can be seen that the ASR method is able to deal with the challenging issue of weak feature extraction in fault diagnosis of blade crack. Compared with the EEMD method, the proposed ASR method is superior to the EEMD method in extracting fault characteristic from blade crack. The study shows that weak characteristic frequency of cracked blade can be extracted gradually from strong noise background owing to the energy transfer mechanism from high-frequency area to low-frequency area.

(2) As the working conditions of centrifugal fan are affected by the fluid and structural field, the vibration transmission paths of blade with crack are complicated, which may lead to deteriorating or attenuating vibration response of fault blade through dissipation and interference effects. In Section 4 , we can find that the time-domain waveforms and the frequency spectra displayed in Figures 8, 10, and 12 do not show useful fault information for impeller fault diagnosis. Thus, the fault characteristic cannot be detected through tradition analysis method and it needs special techniques to analyze the obtained signals. That is why we presented the ASR method to extract weak feature of blade with crack in this paper.

(3) At present, our research is mainly aimed at the crack of blades in an impeller for centrifugal fan fault diagnosis and few people study vibration property owing to multiple cracks in an impeller. However, in practical engineering, the cracks often appear on several blades in the impeller which makes the vibration of the impeller structure more complex. Therefore, it is necessary to adopt special methods to deal with multiple cracks fault in the operation of the fan. The authors are conducting experiments on the fan test rig with multiple cracks fault and will present the results in their future work.

\section{Conclusions}

In this paper, blade crack features of centrifugal fan are pointed out first. Then an adaptive stochastic resonance (ASR) method is adopted to deal with weak characteristic extraction in fault diagnosis of blade crack. At last, a centrifugal fan test rig is established and two cases of faults in the test rig are simulated. The vibration signals collected at the same speed are used to verify the effectiveness of the proposed method. The proposed fault diagnosis scheme based on the ASR method has been applied to the analysis of practical blade vibration signals carrying crack information. In comparison with the EEMD analysis method and the traditional Fourier transform method, the experimental results verify the effectiveness of the proposed method of blade crack fault diagnosis.

\section{Conflict of Interests}

The authors declare that there is no conflict of interests regarding the publication of this paper.

\section{Acknowledgments}

This research is supported by the National Natural Science Foundation of China (51475356 and 51035007) and the National Basic Research Program of China ("973" Program) (no. 2011CB706805). The authors would like to thank the anonymous reviewers for their valuable comments and suggestions.

\section{References}

[1] A. R. Rao and B. K. Dutta, "Vibration analysis for detecting failure of compressor blade," Engineering Failure Analysis, vol. 25, pp. 211-218, 2012.

[2] L. Witek, "Experimental crack propagation and failure analysis of the first stage compressor blade subjected to vibration," Engineering Failure Analysis, vol. 16, no. 7, pp. 2163-2170, 2009.

[3] J. Yan and Y. Xu, "Operational fault feature extraction of blade based on vibration of wind turbine," in Proceedings of the International Conference on Control, Automation and Systems Engineering (CASE '11), pp. 1-3, Singapore, July 2011.

[4] A. A. Gubran and J. K. Sinha, "Shaft instantaneous angular speed for blade vibration in rotating machine," Mechanical Systems and Signal Processing, vol. 44, no. 1-2, pp. 47-59, 2014.

[5] R. J. Kuo, "Intelligent diagnosis for turbine blade faults using artificial neural networks and fuzzy logic," Engineering Applications of Artificial Intelligence, vol. 8, no. 1, pp. 25-34, 1995.

[6] J. M. Barragan, "Engine vibration monitoring and diagnosis based on-board captured data," in RTO AVT Symposium, pp. 811, Manchester, UK, 2001, RTO-MP-079(1).

[7] K. P. Maynard and M. Trethewey, "Blade and shaft crack detection using torsional vibration measurements part 1: feasibility studies," Noise and Vibration Worldwide, vol. 31, no. 11, pp. 9-15, 2000.

[8] K. P. Maynard and M. Trethewey, "Blade and shaft crack detection using torsional vibration measurements part 2: resampling to improve effective dynamic range," Noise \& Vibration Worldwide, vol. 32, no. 2, pp. 27-29, 2001.

[9] K. Maynard and M. Trethewey, "Blade and shaft crack detection using torsional vibration measurements part 3: field application demonstrations," Noise \& Vibration Worldwide, vol. 32, no. 11, pp. 16-23, 2001.

[10] N. E. Huang, Z. Shen, S. R. Long et al., "The empirical mode decomposition and the Hilbert spectrum for nonlinear and non-stationary time series analysis," Proceedings the Royal Society A: Mathematical, Physical and Engineering Sciences, vol. 454, no. 1971, pp. 903-995, 1998.

[11] Z. H. Wu and N. E. Huang, "Ensemble empirical mode decomposition: a noise-assisted data analysis method," Advances in Adaptive Data Analysis, vol. 1, no. 1, pp. 1-41, 2009.

[12] W. Yang, J. Jiang, P. J. Tavner, and C. J. Crabtree, "Monitoring wind turbine condition by the approach of empirical mode decomposition," in Proceedings of the International Conference on Electrical Machines and Systems (ICEMS '08), pp. 736-740, Wuhan, China, October 2008. 
[13] W. X. Yang, R. Court, P. J. Tavner, and C. J. Crabtree, "Bivariate empirical mode decomposition and its contribution to wind turbine condition monitoring," Journal of Sound and Vibration, vol. 330, no. 15, pp. 3766-3782, 2011.

[14] A. Abouhnik and A. Albarbar, "Wind turbine blades condition assessment based on vibration measurements and the level of an empirically decomposed feature," Energy Conversion and Management, vol. 64, pp. 606-613, 2012.

[15] Y. Lei, Z. He, and Y. Zi, "Application of the EEMD method to rotor fault diagnosis of rotating machinery," Mechanical Systems and Signal Processing, vol. 23, no. 4, pp. 1327-1338, 2009.

[16] Y. G. Lei, D. Han, J. Lin, and Z. J. He, "Planetary gearbox fault diagnosis using an adaptive stochastic resonance method," Mechanical Systems and Signal Processing, vol. 38, no. 1, pp. 113124, 2013.

[17] S. Mitaim and B. Kosko, "Adaptive stochastic resonance," Proceedings of the IEEE, vol. 86, no. 11, pp. 2152-2183, 1998.

[18] N. Hu, M. Chen, and X. Wen, "The application of stochastic resonance theory for early detecting rub-impact fault of rotor system," Mechanical Systems and Signal Processing, vol. 17, no. 4, pp. 883-895, 2003.

[19] Q. H. Ye, H. N. Huang, X. Y. He, and C. H. Zhang, "Improved bearing estimates of weak signals using stochastic resonance and frequency shift techniques," in MST/IEEE Oceans Conference Record, vol. 5, pp. 2410-2413, 2003.

[20] Q. H. Ye, H. N. Huang, X. Y. He, and C. H. Zhang, "A study on the parameters of bistable stochastic resonance systems and adaptive stochastic resonance," in Proceedings of the IEEE International Conference on Robotics, Intelligent Systems and Signal Processing, vol. 1, pp. 484-488, Changsha, China, October 2003.

[21] B. Xu, F. Duan, R. Bao, and J. Li, "Stochastic resonance with tuning system parameters: the application of bistable systems in signal processing," Chaos, Solitons and Fractals, vol. 13, no. 4, pp. 633-644, 2002.

[22] Q. He, J. Wang, Y. Liu, D. Dai, and F. Kong, "Multiscale noise tuning of stochastic resonance for enhanced fault diagnosis in rotating machines," Mechanical Systems and Signal Processing, vol. 28, pp. 443-457, 2012.

[23] Q. B. He and J. Wang, "Effects of multiscale noise tuning on stochastic resonance for weak signal detection," Digital Signal Processing, vol. 22, no. 4, pp. 614-621, 2012.

[24] Y.-G. Leng, T.-Y. Wang, Y. Guo, Y.-G. Xu, and S.-B. Fan, "Engineering signal processing based on bistable stochastic resonance," Mechanical Systems and Signal Processing, vol. 21, no. 1, pp. 138-150, 2007.

[25] X. Zhang, N. Hu, L. Hu, and Z. Cheng, "Stochastic resonance in multi-scale bistable array," Physics Letters: A, vol. 377, no. 13, pp. 981-984, 2013.

[26] Y. G. Leng, Y. S. Leng, T. Y. Wang, and Y. Guo, "Numerical analysis and engineering application of large parameter stochastic resonance," Journal of Sound and Vibration, vol. 292, no. 3-5, pp. 788-801, 2006.

[27] B. Li, J. Li, and Z. He, "Fault feature enhancement of gearbox in combined machining center by using adaptive cascade stochastic resonance," Science China Technological Sciences, vol. 54, no. 12, pp. 3203-3210, 2011.

[28] B. Li, J. Li, J. Tan, and Z. He, "AdSR based fault diagnosis for three-axis boring and milling machine," Journal of Mechanical Engineering, vol. 58, no. 9, pp. 527-533, 2012.
[29] J. M. Li, X. F. Chen, and Z. J. He, "Adaptive stochastic resonance method for impact signal detection based on sliding window," Mechanical Systems and Signal Processing, vol. 36, no. 2, pp. 240255, 2013.

[30] R. Benzi, A. Sutera, and A. Vulpiani, "The mechanism of stochastic resonance," Journal of Physics A: Mathematical and General, vol. 14, no. 11, pp. L453-L457, 1981.

[31] M. Ataei and M. Osanloo, "Using a combination of genetic algorithm and the grid search method to determine optimum cutoff grades of multiple metal deposits," International Journal of Surface Mining, Reclamation and Environment, vol. 18, no. 1, pp. 60-78, 2004.

[32] J. Y. Tan, X. F. Chen, J. Y. Wang et al., "Study of frequency-shifted and re-scaling stochastic resonance and its application to fault diagnosis," Mechanical Systems and Signal Processing, vol. 23, no. 3, pp. 811-822, 2009.

[33] R. Zhao, R. Q. Yan, and R. X. Gao, "Dual-scale cascaded adaptive stochastic resonance for rotary machine health monitoring," Journal of Manufacturing Systems, vol. 32, no. 4, pp. 529535, 2013.

[34] J. Tan, X. Chen, Y. Lei, and Z. He, "Adaptive frequency-shifted and re-scaling stochastic resonance with applications to fault diagnosis," Journal of Xian Jiaotong University, vol. 43, no. 7, pp. 69-73, 2009.

[35] H. Li, X. Zhang, and F. Xu, "Experimental investigation on centrifugal compressor blade crack classification using the squared envelope spectrum," Sensors, vol. 13, no. 9, pp. 1254812563, 2013.

[36] A. R. Rao and B. K. Dutta, "Non-intrusive method of detecting turbine blade vibration in an operating power plant," in International Conference on Noise and Vibration Engineering (ISMA '10), Katholieke Universiteit, Leuven, Blegium, 2010.

[37] A. R. Rao, "A method for non-intrusive on-line detection of turbine blade condition," WO2008093349-AI, 2008. 

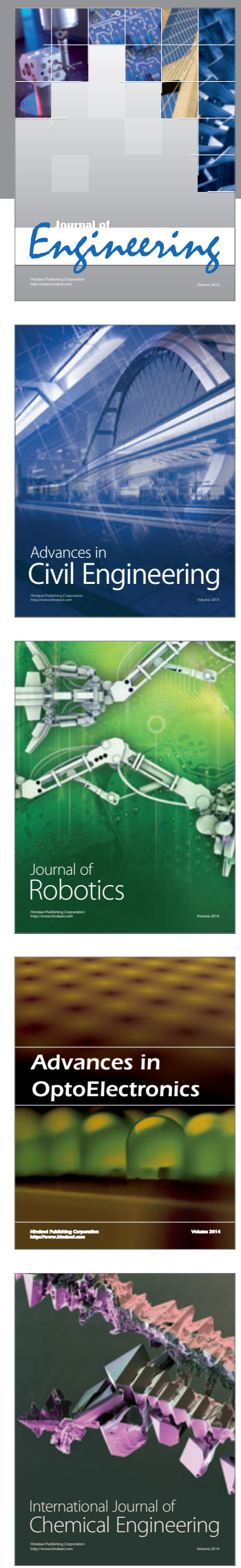

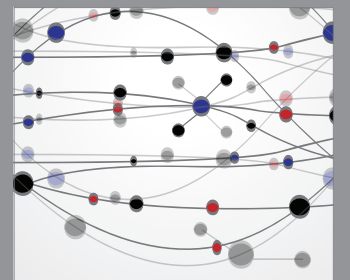

The Scientific World Journal
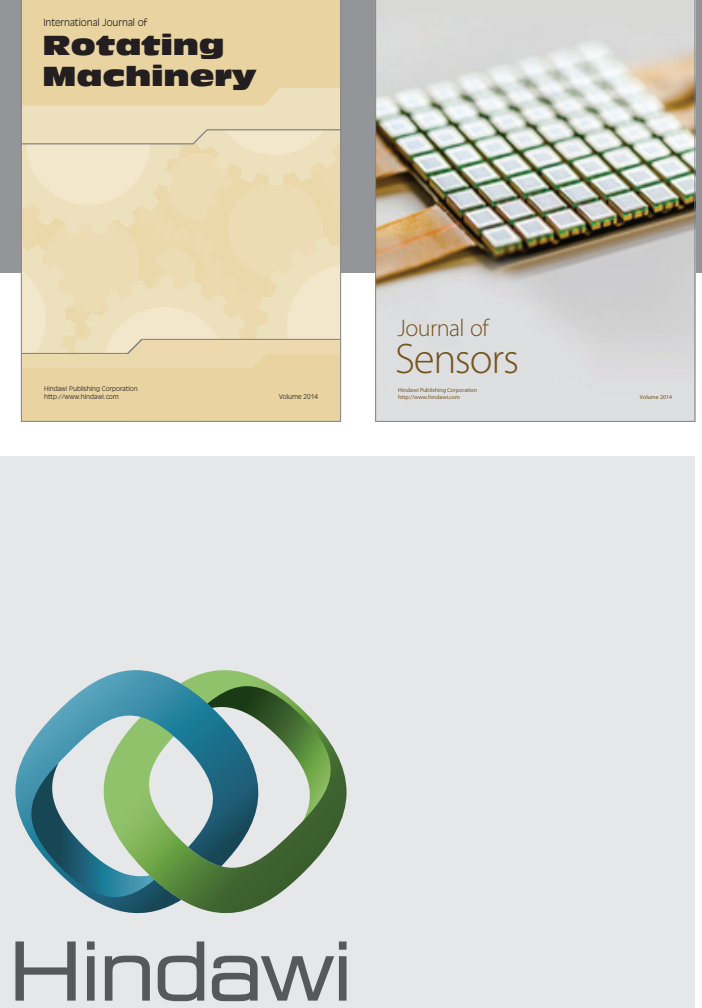

Submit your manuscripts at http://www.hindawi.com
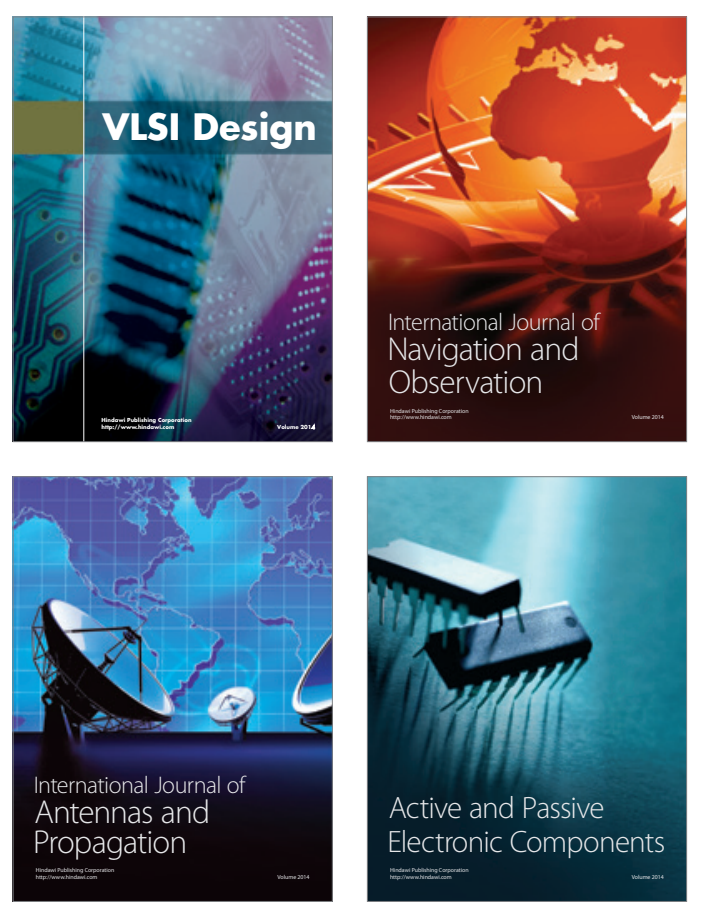
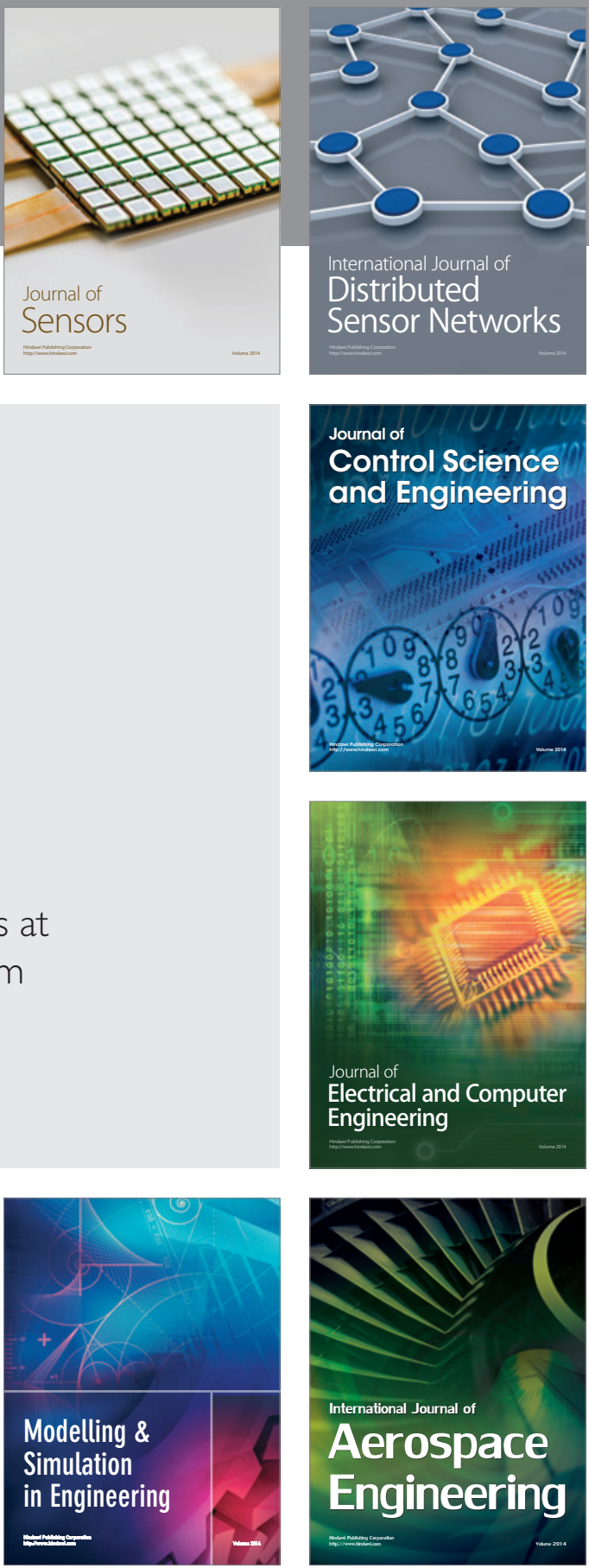

Journal of

Control Science

and Engineering
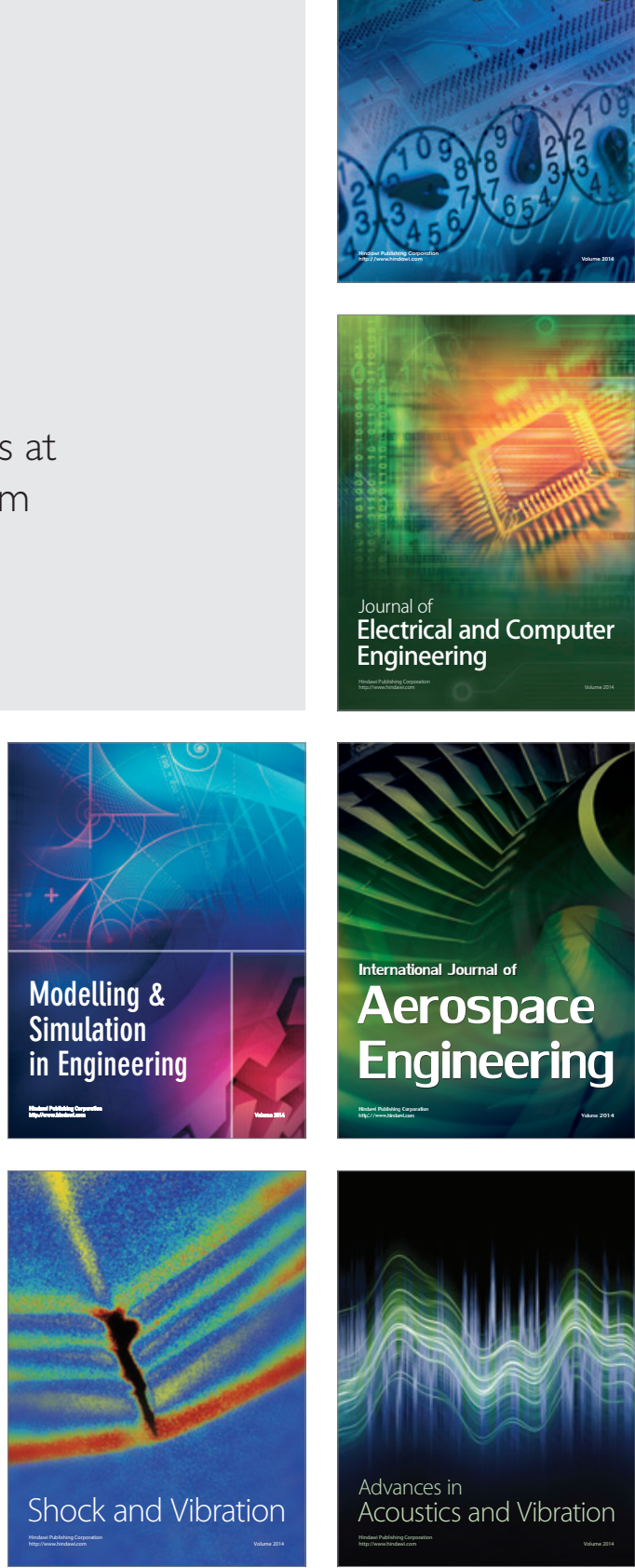